\title{
Le dialogue interdisciplinaire mis à l'épreuve : réflexions à partir d'une recherche sur les blattes urbaines
}

\author{
Nicole Mathieu, Colette Rivault, Nathalie Blanc, Ann Cloarec
}

\author{
C'est en s'appuyant sur des expériences de recherche que l'on sortira du terrain \\ spéculatif le débat sur ce qui doit et peut être l'interdisciplinarité, et que l'on forgera \\ les savoir-faire indispensables pour la mettre en pratique. II en va ainsi avec cette \\ réflexion sur une recherche interdisciplinaire arrivée à son terme et l'évaluation de \\ ses résultats par des chercheurs qui l'ont réalisée.
}

Nicole Mathieu, Nathalle Blanc Laboratoire de géographie, Strates, université de Paris-1. Ura 142 du CNRS, 191, rue Saint-Jacques, 75005 Paris

Colette Rivault, Ann Cloarec Laboratoire d'éthologie de Rennes-I, université de

Rennes,

Ura 373 du CNRS, campus de Beaulieu. 35000 Rennes cedex

1 La ville au risque de l'écologie : questions à l'environnement urbain, ministère de l'Équipement (METT), Plan Urbain, ministère de I'Environnement (SRETIE) 1992, 30 p. (Programme - Écologie urbaine -).

2 En France on trouve principalement trois espèces :

Blatta orientalis L., Blattella germanica (L.) et Supella longipalpa (F.) qui diffèrent par bien des points de leur cycle de développement, de leur biologie et de leur écologie.
En réponse à l'appel d'offres * La ville au risque de l'écologie $\cdot 1$, une équipe associant des éthologistes étudiant l'écologie et l'éthologie d'espèces d'insectes qui vivent uniquement en milieu urbain - les blattes -, à des géographes soucieuses de la réintroduction de la question de la nature dans la géographie urbaine, engagea en 1993 une recherche interdisciplinaire entre sciences sociales et sciences naturelles intitulée " Les blattes en milieu urbain - (Rivault, Cloarec, Blanc, Mathieu, 1995)

Les blattes, insectes d'origine tropicale introduits involontairement dans l'habitat humain, sont devenues, pour certaines d'entre elles, des espèces animales urbaines. Présentes actuellement en France ${ }^{2}$ dans toutes les villes importantes, les blattes sont des espèces omnivores et cosmopolites. Elles sont installées dans tous les types d'habitation : hôpitaux, restaurants, commerces alimentaires, appartements privés... La recherche menée porte uniquement sur l'espèce Blattella germanica qui est de loin l'espèce la plus répandue en France. Cette espèce ne s'est pas adaptée aux différences climatiques, mais a cherché des microclimats favorables correspondant à ses propres exigences. Elle a trouvé dans le milieu urbain sa niche écologique (cf. La niche écologique de la blatte urbaine). Elle vit donc uniquement dans les locaux habités, en particulier dans les immeubles collectifs qui lui assurent les conditions optimales pour son développement. Sa présence et sa pullulation ont conduit dans toutes les grandes villes du monde à la mise en place de politiques de contrôle pour * en débarrasser - les habitants, politiques coûteuses et peu efficaces.

La recherche a été conduite dans trois tours HLM appartenant à l'ensemble de grands collectifs appelé " la Zup Sud ", construit à Rennes entre 1965 et 1975. Ces trois tours, Volga 2, Volclair 14 et Le Strat 1, font partie d'un des quartiers de la Zup, le Blosne, qui, dans le cadre de la politique de la ville, a été inscrit depuis 1989 en DSQ (Développement social de quartiers) du fait de son taux de chômage et d'illétrisme, mais aussi de la dégradation du bâti et de la vie sociale (cf. Les habitants des " 3 tours "). Le problème de pullulation des blattes était posé par les habitants, mais en proportion variable selon les trois tours et à l'intérieur de chacune d'elles, ce qui a justifié leur choix pour l'expérimentation de méthodes de désinsectisation et d'enquêtes auprès des locataires.

Lobjectif n'est pas de présenter ici les résultats de cette recherche qui a dẻjà donné lieu à plusieurs publications (Rivault, Mathieu, Cloarec, Blanc, 1993, 1994, 1995). Nous voulons, en les prenant certes pour appui, tenter plutôt un retour critique sur notre pratique de l'interdisciplinarité du point de vue de son efficacité et de ses retombées théoriques. Cette expérience s'inscrit en effet dans le courant préconisant la recherche interdisciplinaire depuis plusieurs années (Legay, 1986 ; Jollivet dir., 1992). Trois de ses traits rendent - nous semble-t-il - exemplaire la mise à nu ${ }^{3}$ des problèmes posés par l'interdisciplinarité dans le champ de recherche en formation qu'est l'environnement (Jollivet, Pavé, 1993), urbain en particulier.

C'est d'abord le fait que, contrairement à ce qui est souvent prôné dans la plupart des programmes pluridisciplinaires depuis Plozevet ${ }^{4}$, celle ci est à caractère limité : seules deux disciplines ont été mobilisées, l'éthologie et la géographie. C'est sans doute l'une des raisons majeures qui expliquent l'impression de réussite pour l'ensemble des chercheurs qui y ont pris part.

C'est ensuite qu'il s'agit d'une question délibérément finalisée autour d'un cas de figure type, un problème sanitaire qui se pose au niveau mondial (cf.

\footnotetext{
Abstract: This paper is related to an interdisciplinary research (ecology and geography) centred on a very practical social question: how to improve the control of Blattella germanica (coakroaches) which are proliferating in dwellings, thus deteriorating the inhabitants quality of life. Three mainpoints have been emphasized in this research. First, it has been pointed out that it is essential to sort out specific problems inherent to interdisciplinary praxis, together with the means to solve them. Second, the relationship between geography and animal ecology has been analysed in depth; third, interesting scientific and applied results have emerged from this joint research.
} 
Un problème sanitaire damplitude mondiale). Le - problème " à résoudre : le contrôle d'une espèce urbaine et de sa pullulation dans le cadre d'un protocole très précis (Rivault et al., 1995), se situe à l'intersection des sciences du vivant et des sciences sociales. II appelle l'interdisciplinarité. II s'énonce en deux questions pragmatiques clairement définies de part et d'autre. La demande des éthologistes aux géographes est ainsi exprimée : peut-on améliorer l'efficacité des campagnes de désinsectisation en HLM en connaissant mieux les comportements et les pratiques des habitants ? Les géographes ont accepté de répondre à la question principale en la transformant : les représentations que se font les habitants de la blatte et leurs pratiques à l'égard de cet animal sont-elles identiques selon les individus, les familles et les groupes sociaux et jouent-elles un rôle dans la variabilité de l'éradication ?

Le troisième trait réside dans le caractère insolite de cette association. Les géographes étaient-ils les mieux placés pour répondre à cette demande alors que la sociologie des rapports à l'animal a déjà ses lettres de noblesse (Vourc'h, Pelosse, 1988 ; Digard, 1990 ; Vourc'h, 1990 ; Micoud, 1993) ? Plusieurs tentatives pour impliquer certains d'entre eux dans cette recherche ont échoué du fait du caractère affirmé et construit des problématiques portant sur le rapport social à l'animal. Excluant la naturalité de l'animal, les sociologues s'intéressent à la dimension imaginaire de la nature animale ; l'émergence de nouvelles représentations et pratiques à l'égard de certains animaux prend un sens et renvoie à des changements de valeurs et de normes. Contrairement à la sociologie, la géographie admet la matérialité et la naturalité des objets géographiques. Les géographes de l'équipe ont vu dans cette demande des éthologistes l'opportunité de s'impliquer dans une démarche oủ représentations et faits réels étaient confrontés.

Aussi l'engagement dans cette recherche interdisciplinaire a reposé de part et d'autre sur des intérêts hétérogènes : c'était un pari pour les éthologistes que de supposer que les chercheurs des sciences de I'homme, qu'ils soient sociologues, anthropologues ou géographes, leur permettraient de sortir de certaines de leurs impasses ; c'était un parti pris en faveur de l'interdisciplinarité pour des géographes qui en font une condition méthodologique nécessaire à la mise en évidence des interactions entre systèmes naturels et systèmes sociaux ${ }^{5}$.

\section{La niche écologique de la blatte urbaine Blattella germanica $\mathrm{L}$.}

La niche écologique correspond à l'ensemble des paramètres qui caractérisent les exigences écologiques (climatiques, alimentaires, reproductives, etc.) propres à une espèce vivant dans un écosystème (Ramade, 1993).

Blattella germanica, espèce cosmopolite, ne s'est pas adaptée aux différents climats sous lesquels elle se développe, mais $\mathrm{a}$, au contraire, cherché des microclimats favorables correspondant à ses propres exigences écologiques. La plupart des habitations humaines fournissent les conditions que Blattella germanica recherche. Elle y trouve une zone optimale, caractérisée par une interaction des différents facteurs car, finalement elle a besoin des mêmes artifices que l'homme : en permanence des sources d'eau, de nourriture, de chaleur et des abris. Elle trouve là un microclimat relativement stable où les conditions ambiantes telles que l'hygrométrie, la température et la lumière varient peu. Ces conditions sont quasi identiques dans toutes les parties du monde. Dans I'habitation, les cuisines sont les premières zones colonisées. Si la densité de blattes dans une cuisine augmente de manière importante, et que la capacité de charge du milieu est dépassée, les animaux pourront se déplacer et s'installer dans des zones moins favorables. On observera alors une dispersion vers les autres pièces de I'habitation ou même, dans un immeuble collectif, vers un appartement contigu. Dans un immeuble collectif, la distance entre deux zones favorables, entre deux cuisines par exemple (que ce soit dans le sens horizontal ou vertical), est inférieure aux distances que la blatte est capable de parcourir, puisque des expériences de capture-recapture ont montré qu'elle pouvait parcourir des distances allant jusqu'à 86 m (Rivault, 1989, 1990). Dans un habitat pavillonnaire, la distance entre deux endroits favorables devient trop importante et ce milieu ne présente plus la continuité des conditions de milieu requises (température en particulier). C'est une des raisons pour lesquelles on ne trouve des blattes que très rarement dans les pavillons isolés, du moins en climat tempéré.

En conséquence, bien que la blatte soit un animal inféodé strictement au milieu urbain et à la présence de l'homme, elle n'investit pas tout le milieu urbain, n'occupant ni la rue, ni les zones pavillonnaires, ni les parcs. On ne la trouve pas dans certains bâtiments comme des entrepôts où il n'y a pas de nourriture, ni d'eau, ni même de chauffage. Elle se rencontre plutôt dans les restaurants, cantines et autres établissements qui traitent de denrées alimentaires et des habitations privées quel que soit leur standing.

En définitive, on la trouve principalement dans des immeubles collectifs, et dans ces immeubles, dans des appartements occupés régulièrement. La blatte est installée dans un milieu urbain présentant une certaine durée d'habitation. Il s'agit de la partie la plus artificialisée du milieu urbain, là où les variations climatiques et saisonnières sont tamponnées au maximum. Son habitat est toujours associé à l'activité de l'homme et sa capacité de dispersion sur de grandes distances s'est accrue avec l'augmentation de la distance et de la fréquence de nos déplacements. La description de la niche écologique de Blattella germanica a valeur d'exemple. En effet, les autres espèces urbaines ont des exigences écologiques qui sont largement superposables. Ces espèces, tout en occupant un habitat morcelé, ont développé des comportements qui leur ont permis d'atteindre une répartition très large et quelquefois mondiale.

\section{Références}

Ramade F. (1993). Dictionnaire encyclopédique de l'écologie et des sciences de lenvironnement, Paris, Édisciences, $822 \mathrm{p}$.

Rivault C (1989). - Spatial distribution of the cockroach Blattella germanica, in a swimming-bath facility . Entomol. Exp. Appl. 53, 247-255

Rivault C. (1990). - Distribution dynamics of Blattella germanica in a closed urban environment Entomol. Exp. Appl. 57, 85-91 


\section{Les habitants des " 3 tours "}

Les trois tours étudiées se situent dans le quartier du Blosne, dans le sudouest de Rennes, dans une zone appelée la Zup sud. Elles s'inscrivent dans un grand ensemble urbain d'immeubles collectifs, dont une partie importante appartient à un organisme HLM. La population de ce quartier et des trois tours étudiées a des caractéristiques plus marquées sur certains points que lensemble du parc HLM de la ville de Rennes et, a fortiori que les autres quartiers de la ville de Rennes : l'importance des familles nombreuses, la jeunesse de la population (un grand nombre de jeunes adultes vivent en cohabitation avec leurs parents), le nombre d'étrangers (les nationalités les plus représentées sont celles des pays du Maghreb et des pays d'Asie), enfin l'inactivité des ménages et la modestie de leurs revenus. Le cumul des difficultés et plus particulièrement le faible niveau de ressources des habitants les conduisent à demeurer, dans des immeubles à bas loyers, aux formes urbaines rejetées. En effet, l'ensemble des tours du parc HLM du Blosne groupe une proportion importante de ménages qui réside dans le même logement depuis plus de 10 ans et le taux de rotation global sur les groupes est peu élevé, tendance qui se confirme sur l'ensemble du parc de la ville de Rennes. Le regroupement de nombreuses familles cumulant des difficultés contribue à la dégradation des parties communes des immeubles, joue sur la gestion locative (mobilité réduite, impayés...) et sur l'ensemble de la vie sociale de ce quartier (échec scolaire, chômage...). C'est un ensemble urbain ayant des difficultés sociales particulières et une - image - en général négative.

Certains indicateurs révèlent des différences entre les trois tours. La tour A, en particulier, regroupe une proportion plus importante de ménages inactifs, et de familles à faibles revenus touchant une APL (Aide personnalisée au logement : indicateur du niveau de ressources de ménages, en rapport avec la composition familiale et le nombre d'enfants à charge). Ces disparités entre les tours, au sein même des ilots, sont explicables, notamment, par la répartition des logements de grande taille ou de petite taille, mais surtout par le mode de financement de construction de la tour qui entraine un loyer plafonné selon les ressources. La tour A, qui a fait l'objet à l'origine d'un mode de financement différent des autres tours pour sa construction, bénéficie donc de niveaux de loyers plus faibles et regroupe des personnes aux ressources moindres. De manière générale, même si ces trois tours ont des caractéristiques plus marquées sur certains points que l'ensemble du parc HLM de la ville de Rennes, aucune n'est, dans l'ensemble du parc HLM du Blosne, celle qui regroupe le plus d'indicateurs au dessus de la moyenne.

Occupation sociale de trois tours du parc HLM du Blosne (1989)

\begin{tabular}{|c|c|c|c|c|c|}
\hline & $\begin{array}{c}\text { Nombre } \\
\text { de logements }\end{array}$ & $\begin{array}{c}\text { Nombre } \\
\text { de personnes }\end{array}$ & \multicolumn{2}{|c|}{$\begin{array}{c}\text { Ménages constitués } \\
\text { d'une personne seule (en \%) }\end{array}$} & $\begin{array}{l}\text { Vombre de personnes } \\
\text { par logement }\end{array}$ \\
\hline Tour A & 94 & 237 & \multicolumn{2}{|l|}{37} & 2,8 \\
\hline Tour B & 94 & 282 & \multicolumn{2}{|l|}{33} & 3,1 \\
\hline Tour C & 96 & 288 & \multirow{2}{*}{\multicolumn{2}{|c|}{31}} & 3,1 \\
\hline Parc HLM & & & & & \\
\hline \multirow[t]{2}{*}{ du Blosne } & 1306 & 4300 & \multicolumn{2}{|l|}{26} & 3,2 \\
\hline & $\begin{array}{l}\% \text { ménages } \\
5 \text { enfants }\end{array}$ & $\begin{array}{l}\text { \% logements } \\
\text { surpeuplés }\end{array}$ & $\begin{array}{l}\text { \% familles } \\
\text { monoparentales }\end{array}$ & $\begin{array}{l}\% \text { de moins } \\
\text { de } 18 \text { ans }\end{array}$ & $\begin{array}{l}\text { \% personnes } \\
\text { étrangères }\end{array}$ \\
\hline Tour A & 4 & 10 & 17 & 31 & 33 \\
\hline Tour B & 9 & 13 & 18 & 37 & 33 \\
\hline Tour C & 11 & 13 & 16 & 38 & 30 \\
\hline $\begin{array}{l}\text { Parc HLM } \\
\text { du Blosne }\end{array}$ & 7 & 13 & 19 & 35 & 33 \\
\hline
\end{tabular}

aDéficit de deux chambres (ratio : une chambre par enfant et une pour le ou les parents)

\begin{tabular}{lcccc}
\hline & $\begin{array}{c}\text { \% ménages } \\
\text { inactifs }\end{array}$ & $\begin{array}{c}\text { \% revenus ménages } \\
\text { inférieurs a } 20000 \mathrm{~F}\end{array}$ & $\begin{array}{c}\text { \% ménages } \\
\text { touchant I'APL }\end{array}$ & $\begin{array}{c}\text { \% ménages de plus } \\
\text { de 10 ans dans la tour }\end{array}$ \\
\hline Tour A & 48 & 47 & 63 & 32 \\
Tour B & 32 & 26 & 48 & 33 \\
Tour C & 42 & 27 & 56 & 34 \\
Parc HLM & 31 & 32 & 54 & 38 \\
du Blosne & & & \\
\hline
\end{tabular}

(Source : Analyse du peuplement, OPHLM de la Ville de Rennes, Mission de maitrise đ’œuvre sociale sur le quartier du Blosne, février 1992,93 p.) 
Les questions théoriques sous-jacentes à cette recherche peuvent même apparaître à la limite de la divergence : dans la mesure où l'homme fait partie de l'écosystème des populations animales qualifiées d'espèces urbaines, comment l'éthologie peut penser et énoncer la relation homme-animal se demandent les premiers ? Comment réintroduire à part entière la dimension naturelle de la ville dans la géographie urbaine ? pourquoi fait-on si peu de cas des représentations de la nature en ville et de la matérialité naturelle de la ville dans la prise en compte de la crise urbaine, se demandent les seconds ? Certes les deux équipes ont quelques interrogations en commun : qu'est-ce que l' e écologie urbaine ${ }^{6} 6$ ? comment traiter les interactions entre le social et le biologique ? Elles partagent aussi certaines hypothèses fortes : la nécessité d'introduire dans l'idée de qualité de vie en ville la dimension naturaliste, les rapports des habitants à la nature. Mais ces points communs suffisent-ils pour surmonter le décalage des enjeux au départ ?

On engagera dans une première partie la réflexion sur les problèmes que cette recherche a posés : comment ils ont été identifiés et définis, comment ils ont été résolus ou surmontés, ce dont on rend rarement compte avec précision. Dans la deuxième partie, on s'appuiera sur les résultats les plus significatifs pour montrer les apports spécifiques de l'interdisciplinarité et illustrer la valeur heuristique de notre pratique interdisciplinaire. Enfin on conclura sur notre réponse à la question finalisée qui est à l'origine de la recherche.

\section{Deux types de problèmes, des moyens pour les surmonter}

\section{La distinction des cultures scientifiques}

Cette position entre nécessité et opportunité, entre intuition et pari permet de déceler un premier type de problèmes qui tient au jeu de rôles que la pratique de l'interdisciplinarité provoque entre les chercheurs de disciplines distinctes.

Si l'on dit jeu de rôles, c'est parce que, au début de ce programme, le regard que portait chaque groupe de recherches sur l'autre était tout à la fois fait d'attente et de suspicion. Les caractéristiques des deux démarches semblaient divergentes. L'exigence de mesure et de démonstration paraissait plus affirmée chez les écologues même si la quantification, l'expérimentation et l'analyse multifactorielle (Rivault, Cloarec, 1995) n'éliminent pas la complexité des phénomènes. La présentation des résultats réunis - pour tenter d'expliquer la présence-absence de blattes en relation avec les conditions du milieu . devait suivre les règles de la communauté scientifique (les sciences de la vie) qui les valident. Les éthologistes s'attendaient à ce que les résultats des sciences sociales leur permettent d'introduire le " facteur humain - sous forme d'indicateur (sexe, taille du logement, densité d'habitants par mètre carré, nationalité française ou étrangère, statut social...) de la même manière que les appartements (le milieu des blattes) ont été " notés - par des indicateurs de surface, d'encombrement et de " propreté . II leur semblait que pour démontrer que les représentations et les pratiques des habitants des tours jouent un rôle dans l'efficacité des désinsectisations, il fallait pouvoir mesurer ces pratiques et ces représentations, en établir une typologie et en modéliser les relations avec la dynamique des populations de blattes. Le savoir acquis par expérience sociale ${ }^{7}$ ne paraissait avoir de valeur que dans le domaine limité des avis à dire d'expert. Dans ce premier temps de la recherche, pour les écologues qui abordent le vivant en ignorant implicitement la subjectivité, l'homme-sujet, la démarche des géographes, découvrant la violence et la diversité des discours sur les * cafards $=8$, semblait qualitative et littéraire même si ces derniers s'orientaient vers l'analyse des subjectivités, de la diversité et de la singularité des pratiques et des représentations, en les quantifiant

$\mathrm{Au}$ départ du programme, les géographes de l'équipe se donnent certes les moyens de répondre à la question posée par les méthodes de mesure qui caractérisent leur discipline, en particulier l'analyse démographique localisée. La population des trois tours est analysée pour dégager les différences sociales d'occupation et est confrontée à celle du quartier DSQ et de l'ensemble de la Zup. Des indicateurs auxquels les écologues de l'équipe n'avaient pas pensé, comme l'origine rurale et régionale des habitants, sont testés comme discriminant les attitudes. Une cartographie précise des logements par étage des trois immeubles et de leur occupation est réalisée pour préciser la dimension * humaine - du - milieu - de la blatte. Pourtant, dans ce premier temps, les géographes portent un regard perplexe sur l'exigence de "simplicité - et de démonstration des éthologistes. La vision simplifiée qu'ont les écologues de la population humaine, l'abstraction de leur concept d'homme toujours ramené à un facteur, l'étroitesse de leur notion de milieu qui se limite à la niche des blattes sont perçus comme des obstacles au dialogue. II est vrai que la question qui est posée par les éthologistes les pousse au contraire à privilégier le sujet et la subjectivité, à développer une approche de type anthropologique qui n'était pas prévue au départ de la recherche. Certes les géographes mesureront des pourcentages de réponses au questions posées dans les entretiens. Mais, on y reviendra, les résultats les plus innovants ont été apportés par la partie non directive et la surprenante * liberté verbale * dont font preuve les enquêtés quand on les interroge sur leurs attitudes et conduites par rapport aux cafards. C'est dans ces entretiens, essentiels pour identifier des types de logiques et de décalages entre les représentations et les pratiques, que l'hypothèse d'un lien de causalité entre les pratiques des habitants et l'efficacité de la désinsectisation est apparue. Pour présenter des modèles de rapports habitants/cafards, il a fallu en passer par la méthode des portraits d'habitants et de familles et par la biographie de leurs lieux de vie (cf. Entretien avec une habitante). Face à l'éthologie qui fait un va-et-vient
${ }^{3}$ En tentant d'aller encore plus loin que dans la troisième partie - Questions et méthodes . de l'ouvrage dirigé par Marcel Jollivet (Jollivet dir., 1992), en particulier par rapport à lanalyse de Monique Barrué-Pastor - Linterdisciplinarité en pratiques *, ceci grâce à une autoréflexion équivalente à celle menée par Nicole Mathieu de façon biographique (Mathieu, 1989) ou à propos du programme Piren Causses/Cévennes (Mathieu, 1987).

\section{${ }^{4}$ II s'agit du premier programme} pluridisciplinaire lancé par la DGRST au début des années 1960 sur une commune du Finistère Sud. Depuis, les programmes interdisciplinaires conduits par le CNRS ont généralement confié des programmes à des équipes comportant plusieurs disciplines.

${ }^{5} \mathrm{Cf}$. laxe de recherche de Strates - Interaction systèmes

naturels-systèmes sociaux ; échelles, catégories spatiales et problèmes

d'environnement - in Une charte en trois axes de recherche $(\mathrm{N}$. Mathieu, $\mathrm{C}$ Paix, eds) (1992-93). Strates 7, 105-124.

${ }^{6}$ C'est au Colloque national d'écologie urbaine organisé par JeanMarie Legay à Mions en 1991 (université ClaudeBernard Lyon-I, 1992) qu'est née la volonté de confronter la définition émergente de cette notion pour les sciences du vivant et l'écologie politique, à la définition antérieure par les sciences sociales et l'École de Chicago. 
entre l'observation de terrain et l'expérimentation en laboratoire, les sciences sociales paraissent inachevées comme le dit Morin (Morin, 1996) et l'on imagine mal (même si au contact des éthologistes nous en avons parfois rêvé) de monter une expérience en vraie grandeur avec des protocoles pour vérifier les pratiques des habitants à l'égard de la blatte, le jour comme la nuit et dans les différentes pièces de l'appartement.

En somme, malgré l'acceptation de la division des rôles, les formations disciplinaires, avec leurs codes de méthodes, constituent - on l'a souvent souligné des obstacles à l'interdisciplinarité. Chaque discipline propose des objets qui sont des boîtes noires pour l'autre, les blattes pour les géographes, les hommes pour les éthologistes. Les uns comme les autres, attachés à leur culture scientifique, appréhendent le fait que la pratique interdisciplinaire conduise à une banalisation par un transfert analogique des méthodes de chaque discipline.

La pratique de l'interdisciplinarité modifie les regards que chacun porte sur l'autre quand il y a curiosité scientifique et respect. Dans cette expérience, la taille réduite de l'équipe, entièrement féminine, a favorisé la reconnaissance mutuelle des compétences de chaque discipline. L'exercice a été mené en refusant toute hiérarchisation et instrumentalisation entre les chercheurs ${ }^{9}$. Mais la qualité des rapports entre chercheurs ne permet pas à elle seule de surmonter ce problème de fond entre des sciences qui se pensent et se veulent * exactes - et les sciences de l'homme et de la société. II faut aussi trouver des moyens durables de le dépasser.

\section{Les chemins du dialogue}

A l'actif de cette recherche, il nous semble possible d'avancer deux exemples de ces moyens permettant de fonder un dialogue soutenu. C'est d'abord la construction de références communes rendant possible le contrôle réciproque des connaissances même si elles sont de nature différente. C'est surtout un constant recours à la question finalisée qui requerrait l'interdisciplinarité.

Le choix en commun de ce qu'on peut appeler le terrain est certainement le premier et le plus élémentaire. Dans le cas de cette recherche, les références communes ont été élaborées grâce à une pratique de l'investigation strictement superposée dans les mêmes lieux : les trois tours HLM, les familles et les appartements connus par les chercheurs des deux disciplines. Certes, dans une tour, dans un appartement, les géographes ne voyaient pas le même " paysage ", le même " milieu * ou " environnement * que les éthologistes (nous reviendrons sur la similitude apparente des concepts employés par les deux disciplines). Certes, les données recueillies n'étaient pas les mêmes, ni dans la forme, ni dans le fond. Mais le fait que les chercheurs se référaient avec exactitude aux mêmes espaces, aux mêmes familles, finissait par leur donner la même expérience, validait la confrontation de leurs données et de leurs interprétations. Peu à peu, des notions, comme celle de saleté par exemple, se recomposaient, mêlant le versant objectif du point de vue des risques de pullulation : l'encombrement, le rythme d'évacuation des ordures ménagères, et le versant subjectif, voire fantasma-

\section{Un problème sanitaire d'amplitude mondiale}

Les blattes sont présentes depuis fort longtemps sur terre. Les premiers fossiles, qui présentent une forme générale du corps très proche de celle des blattes vivantes actuellement, datent du carbonifère. La grosse majorité des espèces de blattes actuelles ne sont pas * domestiques -. Parmi les 3500 espèces de blattes connues, 50 espèces seulement occupent des habitats humains. Leur distribution est mondiale : de l'Arctique à I'Antarctique en passant par les Tropiques. Elles se sont répandues en suivant les routes commerciales maritime, terrestre ou aérienne. Leur présence a d'abord été

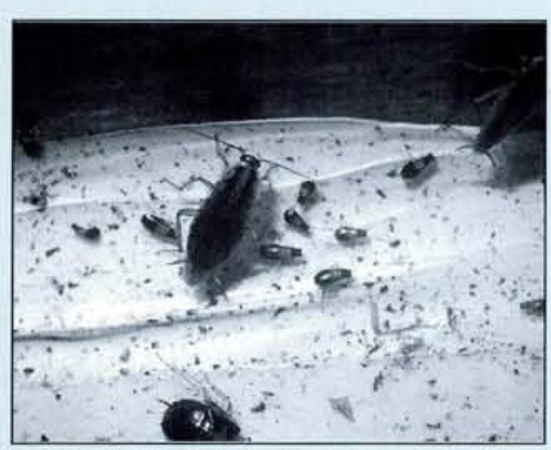

La blatte germanique, Blattella Gemanica $\mathrm{L}$ signalée dans des villes portuaires, puis ensuite dans les villes importantes. Elle dépend donc de l'opportunité des introductions et des dispersions passives liées à I'homme.

Leur statut d'insecte urbain et * nuisible - est relativement récent. Les blattes peuvent déclencher des phobies, des peurs incontrôlées et un malaise social. Elles peuvent également provoquer des allergies ou des formes d'asthme chez certains sujets. Enfin, elles transportent de manière passive des bactéries, des vers, des champignons, des virus et des protozoaires susceptibles d'entraîner, par exemple, des empoisonnements alimentaires et autres formes d'infection (Roth et Willis, 1957). Une étude menée à Rennes a permis d'identifier au moins 56 espèces de bactéries transportées par des blattes dans des bâtiments publics divers (hôpitaux, restaurants, appartements privés et piscines). Parmi ces bactéries se trouvent des espèces pathogènes comme des salmonelles, des entérobactéries et le genre Pseudomonas (Rivault et al., 1993a et b). Dans la mesure oủ les blattes ne sont pas un vecteur obligatoire pour ces espéces, leur rôle exact dans la dissémination d'agents pathogènes est rarement défini avec précision. Le fait qu'elles se nourrissent aussi bien de fèces humains que de nourri- ture humaine indique la possibilité qu'elles transmettent des maladies et donc leur rôle dans les problèmes de santé.

Les blattes, par leur distribution mondiale, leur présence fréquente en milieu urbain et le risque qu'elles présentent pour la santé humaine, font l'objet de tentatives de contrôle par différents moyens dont les traitements insecticides sont les plus fréquents. Malgré les progrès techniques réalisés au niveau de l'efficacité des produits insecticides utilisés, on constate toujours l'échec des campagnes de contrôle des blattes. Lanalyse des facteurs contribuant à l'échec des ces traitements a conduit à réaliser ce travail interdisciplinaire.

\section{Références}

Rivault C, Cloarec A., Le Guyader A. (1993a). · Bacterial load of cockroaches in relation to urban environment ? Epidem. Inf. 110, 317-325

Rivault C., Cloarec A., Le Guyader A. (1993b). * Bacterial contamination of food by cockroaches : J. Environmental Health 55, 21-22

Roth L.M., Willis E.R. (1957). - The medical and veterinary importance of cokroaches : Smithson Misc Collect., 134, 1-147 


\section{Entretien avec une habitante}

Une jeune femme brune, 27 ans, dynamique et plutôt forte, habillée en jogging nous accueille. Nous nous asseyons dans la salle à manger, derrière une grande table rustique en bois. Lappartement est propre et clair, les fenêtres sont ouvertes sur l'extérieur, l'air est frais. Cette femme, ancienne secrétaire de direction d'une association, est mariée à un chauffeur routier. Elle a quatre enfants et bénéficie actuellement d'un congé parental de trois ans qui lui permet de rester chez elle et de s'en occuper. Elle vient de la campagne (Dordogne), qu' elle regrette, et n'a accepté de venir en ville que pour suivre son mari qui avait trouvé du travail à Rennes: - Je ne voulais pas vivre dans une ville, et surtout pas dans une tour. - Elle a décidé de s'adapter à sa nouvelle situation mais désire, à terme, quitter le quartier et la ville : “ Tout gêne ici... mais cest la conjoncture actuelle qui veut ça. Si en plus on fait le choix d'avoir beaucoup d'enfants. on se retrouve là avec des petits salaires, c'est évident. " Elle voit son mari le week-end et reste seule avec ses enfants la semaine, dans un environnement qu'elle juge difficile. Quand elle a emménagé dans l'appartement, les blattes pullulaient (importance de l'histoire résidentielle de l'appartement) mais, progressivement, elle est parvenue à s'en débarrasser complètement. Pour elle, les blattes et les tours sont associées. D'ailleurs, dès le début, elle identifie les blattes car elle se doute que - dans les immeubles comme ça, il pouvait y en avoir, donc on a conclu que ça devait être ça -. Le sentiment que lui inspirent les blattes est mis en relation avec la vie qu'elle menait auparavant à la campagne : "Ce n'est pas de la peur. C'est vrai que je n'avais jamais été confrontée à ça, je n'ai jamais vécu dans un milieu comme ici. On vivait à la campagne, et on était dans un milieu privilégié, on est arrivé ici, c'était de la répugnance ", et - $A u$ départ, jassociais ça à la saleté " Ses représentations de la blatte sont donc identiques à celles des autres habitants : “ Ce n'est jamais agréable de voir des bêtes grouiller chez soi, puis bon ça se situe particulièrement dans la cuisine, là oủ il y a les aliments - mais, depuis lors, elle a réfléchi à ses représentations liant la saleté à la blatte grâce aux informations données notamment par les éthologues pendant les désinsectisations : " On voit quatre mouches dans sa cuisine, on n'a pas peur. On voit quatre blattes, on panique tout de suite. Penser que c'est sale? Non, maintenant, je sais que ce n'est pas sale... Bon, si, c'est sale car il y a toujours de petites crottes. Mais c'est pas plus sale qu un autre insecte. "Mais à la différence d'autres personnes des immeubles, elle met en œuvre de nombreuses pratiques pour éliminer ces insectes. Dès son emménagement, elle a utilisé des insecticides dans les endroits où elle a remarqué qu'il y en avait souvent. Outre cela, ayant observé les mœurs nocturnes des blattes, elle explique : " Il faut aller le soir alors. $\dot{A}$ un moment on tendait des guet-apens, on éteignait la lumière, on revenait une heure après : celles quion voyait, on "pschitait" dessus. " Comme la majorité des personnes interrogées, elle pense que les habitants qui n'ouvrent pas leurs portes aux désinsectiseurs sont responsables de la présence des blattes dans l'immeuble. *Il faut faire du forcing chez toutes les personnes. C'est bien beau de traiter les gens qui veulent bien admettre quils en ont, mais il y a encore des gens qui prennent ça pour un sujet tabou. Avoir des blattes chez soi, ils associent ça à un signe de saleté. - En traitant, elle est arrivée à les éradiquer : les autres sont donc responsables si les blattes reviennent.

Depuis novembre 1991, date de son installation, elle explique qu'elle a lutté pour s'installer véritablement dans cet habitat. En effet, ses amis n'ont pas tous réagi favorablement à la vue du quartier : " Les gens n'étaient pas habitués à nous voir évoluer dans un cadre comme ici, c'est là quion fait le tri de ses amis. - C'est pareil pour l'immeuble. Les gens quand ils rentrent ici, me disent : tu as vu où tu habites, tu as vu comment ça sent, tu as vu de quelle couleur les gens sont. Moi c'était clair, je leur ai dit : il y a des cafards, si ça vous gêne, vous partez, si ça ne vous gêne pas, vous restez. "Mais elle a du lutter également contre l'intolérance et l'indifférence des gens du voisinage : “C'est vraiquau départ javais pris un peu l'habitude de dire : finalement, il ne faut pas regarder ce quill y autour, il faut rentrer chez soi, s'enfermer. C'est chez toi que ça compte, c'est pas à l'extérieur et puis non, en fait il y a des gens très valables ici. C'est vrai qu'il faut faire le tri mais ce qui choque le plus, c'est l'intolérance : c'est toujours la faute à l'autre, ils ne

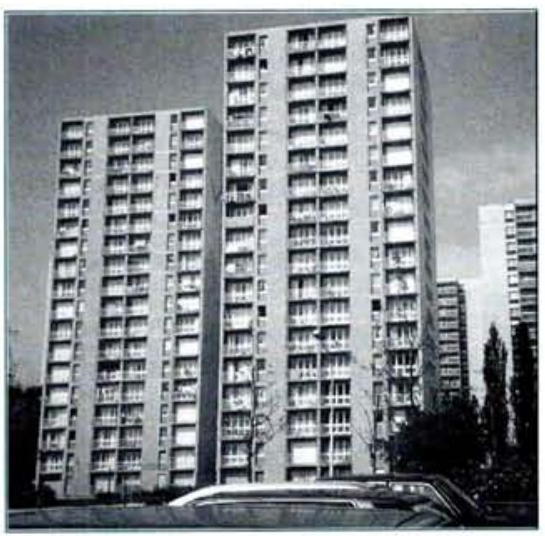

Immeubles ayant fait l'objet de l'étude, situès à Rennes.

prennent jamais rien en charge, ils ne prennent pas leurs responsabilités. " La lutte qu'elle mène dans ce sens est liée à la chasse qu'elle conduit contre les cafards : " Les blattes font partie des choses auxquelles je ne me suis pas faite, il y a d'autres choses pour lesquelles je suis plus tolérante. - Ses pratiques envers la blatte s'inscrivent donc bien dans le cadre d'une lutte plus générale pour s'ajuster à un lieu qui représente la - zone ", et à des pratiques que certains habitants déploient (ignorance mutuelle, irresponsabilité...). De manière générale, il y a une relation entre les modes d'habiter et les pratiques de contrôle de la blatte. Les personnes à même de négocier avec leur mode d'habiter actuel sont peutêtre plus actives pour se débarrasser des blattes. Une partie de la relation homme-blatte peut être expliquée par l'acceptation et la maîtrise ou le rejet du mode d'habiter actuel.

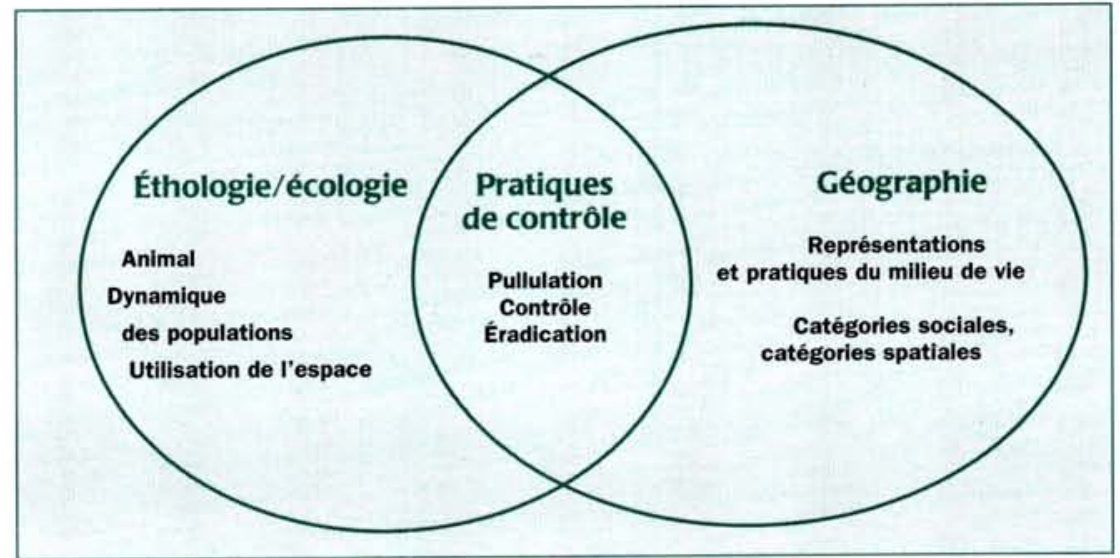

Schéma 1. Lìnterdisciplinarité en schéma. 
tique : la saleté imaginaire du cafard, la saleté des HLM, habitat qu'on n'a pas choisi, la saleté des autres... Les géographes de l'équipe ont redécouvert une proximité avec l'anthropologie, confirmé l'intérêt d'étudier l'homme-habitant en allant jusqu'à l'individu. Les éthologues ont admis l'importance de l'analyse individuelle et de l'approche anthropologique du sale et du propre. Peu à peu s'est imposée la qualité d'une démarche qui croise des résultats issus de l'analyse écologique (nombre de blattes compté après chaque désinsectisation, caractéristiques de la population, produits utilisés, nombre de passages, refus...) avec les discours, les représentations et les pratiques des interviewés.

Plus efficace encore pour résoudre les oppositions de méthodes est de se donner une obligation commune, celle de répondre à la question finalisée à l'origine de l'interdisciplinarité. C'est en s'attachant à cette question : " comment améliorer le contrôle de la pullulation ? ", que chacune des disciplines prend la mesure de ses limites et de la nécessité des complémentarités. Le dialogue se concentre alors sur les connaissances indispensables pour traiter le problème finalisé quelles que soient les méthodes pour les établir. Ainsi les éthologistes prennent conscience que la réponse à l'amélioration de l'efficacité des désinsectisations se trouve plutôt dans la partie qualitative de la démarche des sciences sociales confrontant représentations et pratiques dans la singularité des familles et des individus habitant l'immeuble. L'analyse du processus de décision complexe des acteurs intervenant dans la politique de désinsectisation s'introduit dans la problématique. De même les géographes comprennent que l'appréciation du poids des représentations et des pratiques sur la dynamique des populations de blattes ne peut être validée sans une confrontation avec le protocole construit par les écologues de l'équipe sur la base de leurs travaux antérieurs et sur le suivi rigoureux de l'expérience. L'espace d'intersection des disciplines, le contrôle de la dynamique des populations animales (cf. schéma), est peu à peu cerné. II légitime en retour les méthodologies disciplinaires spécifiques.

\section{Les fausses passerelles des mots communs}

Le deuxième problème concernant le dialogue interdisciplinaire provient paradoxalement, et contrairement au problème précédent, des points de convergence entre les deux disciplines ${ }^{10}$, des notions communes qu'elles manient, celles précisément qui ont motivé leur association. Apparemment identiques,

\section{Analyse multifactorielle des résultats de la campagne de désinsectisation}

Nous avons appliqué une méthode utilisée en écologie pour l'étude des relations espèces-milieux, pour tenter d'expliquer la présence-absence de blattes en relation avec les conditions du milieu. L'analyse factorielle des correspondances avec variables instrumentales (AFCVI) (Lebreton et al., 1991) traite un tableau dans lequel un ensemble d'appartements est décrit par deux groupes de variables : les densités de blattes dans chaque appartement, d'une part, et les facteurs environnementaux dans ces mêmes appartements, d'autre part (Rivault et Cloarec, 1995).

Le premier groupe de variables comprend pour chaque appartement, l'effectif de blattes qui est une variable quantitative que nous avons codée en cinq niveaux. Le niveau 1 correspond à un appartement traité où nous n'avons pas vu de blattes; le niveau 2 , à un appartement traité où nous avons vu quelques individus (moins de 10) ; le niveau 3 , à un appartement traité où nous avons vu une population bien installée (10 à 50 individus) ; le niveau 4 , à un appartement traité où il y avait une pullulation (plus de 50 individus) ; et le niveau 5 à un appartement non traité lors d'une série de traitements.

Le second groupe de variables comprend les variables environnementales et humaines liées à chaque appartement et qui comprennent l'immeuble, le numéro et l'étage, la nationalité des locataires, la durée d'occupation, le nombre d'habitants/100 $\mathrm{m} 2$, la réaction de locataires visà-vis des blattes et le degré de propreté. Les deux premiers axes de l'analyse extraient $48 \%$ de la variabilité totale (Rivault et Cloarec, 1995). Sur le graphe formé par les axes 1 et 2 de l'analyse, les centres de gravité des points qui figurent les différentes densités de blattes observées au cours de cette campagne de désinsectisation présentent un gradient le long de l'axe 1, qui oppose absence de blattes à présence de blattes. Laxe 1 peut donc être qualifié d'axe de présence-absence de blattes. Laxe 2 est difficile à interpréter en terme de densité de blattes.

Lanalyse des facteurs environnementaux sur le graphe formé par ces deux mêmes axes 1 et 2 , montrent que les trois facteurs : immeuble, nombre d'habitants/100 $\mathrm{m} 2$ et propreté, contribuent le plus à la signification de l'axe 1. Ces trois facteurs peuvent être assimilés à une variable composée qui résume les variations des données environnementales que nous avons mesurées dans les appartements où les blattes sont présentes. L'axe 2 peut être interprété comme un axe de propreté puisqu'il oppose les appartements en fonction de leur niveau de propreté.

Nous savons que ces deux axes 1 et 2 sont orthogonaux ; l'orthogonalité de ces axes signifie que les variables représentées par ces deux axes varient de manière indépendante. Dans ce cas, la variable présence-absence de blattes, représentée sur l'axe 1 varie indépendamment de la variable propreté, représentée sur l'axe 2 . Un tel résultat signifie que la présenceabsence de blattes n'est pas liée à la saleté dans les appartements. En revanche, quand elle sont installées, la saleté favorise l'augmentation de la taille de la population.

\section{Références}

Lebreton J.D., Sabatier R., Banco G., Bacou A.M. (1991) - Principal component and correspondence analyses with respect to instrumental variables: an overview of their role in studies of structure-activity and species-environment relationships *, in : Applied multivariate analysis in SAR and environmental studies (J. Devillers, W. Karcher, eds), Luxembourg : ECSC, EEC, EAEC 85-114

Rivault C., Cloarec A. (1995). Limits of insectide cockroach control in council flats in France. $J$. Environ. Management 45, 379-393 
au départ utilisés comme s'ils avaient le même sens, ces mots sont en fait de véritables faux-amis, supports de consensus flous, voire même de contresens. Notre pratique de l'interdisciplinarité conduira à les déconstruire pour dégager des concepts opératoires.

Ainsi, avec le concept de * blattes urbaines - et le recours à l'expression de " qualité de vie en ville ", les éthologistes se réfèrent constamment à la notion de ville, milieu artificiel, opposé à celui de milieu naturel (campagne, forêt, lac...). Mais la ville des écologues n'a rien à voir avec la ville des praticiens et des disciplines de sciences sociales. Leur terme d'urbain est un qualificatif global, de sens commun, et dont le contenu n'a pas besoin d'être défini (Pontier, 1992). La blatte est une espèce dite urbaine parce qu'elle vit dans l'habitat * urbain " de l'homme et plus particulièrement dans des immeubles collectifs où ses déplacements limités sont compatibles avec son installation et son développement. Les écologues font aussi un usage particulier du terme de " campagne - ou de * rural - qui renvoie aussi à l'échelle de l'habitat de la blatte. Ainsi, les blattes ne sont pas une - espèce rurale - dans la mesure oủ il a été prouvé qu'elles se développent plus difficilement dans des habitats pavillonnaires, dans un habitat dispersé, dans les villages groupés de morphologie traditionnelle (maisons, petits immeubles et jardins), ceci pour les deux raisons : l'espèce installée se déplace dans un faible rayon (de l'ordre d'une pièce) et craint le froid ; il y a donc un meilleur contrôle de leur dynamique en dehors des immeubles collectifs et là où le tissu de I'habitat est rendu discontinu par des * espaces naturels * Certains écologues utilisent aussi la notion de ville dans un autre sens, celui de la municipalité (Rennes), mais là encore c'est une notion très peu définie qui ne recouvre que l'aire de compétence de l'office de HLM et de sa politique.

Face à l'usage du terme urbain par les écologues, les géographes de l'équipe prennent la mesure à la fois de la valeur et de la faiblesse de leurs catégories spatiales. Ils disposent certes d'outils plus précis et plus complexes pour décliner la ville et la campagne : espaces urbains, espaces ruraux, types de villes, quartiers, morphologie urbaine, etc. Mais ils découvrent aussi l'inadéquation de ces concepts quand le problème à traiter implique la relation des urbains à un insecte. L'entrée dans l'analyse de la * qualité de la vie en ville - par l'animal remet en cause la définition consensuelle de la ville dans les sciences sociales, invalide l'idée même de ville comme * espace légitime - de l'urbanité opposée à la ruralité (Mathieu, 1996). Elle impose de resserrer l'analyse sur l'habitat précis de la Zup Sud de Rennes et de prendre en compte les niveaux d'organisation et les échelles.

Le problème a été surmonté en commun par une redéfinition précise des échelles de travail, les géographes faisant l'effort de conduire leur analyse à une échelle qui n'est pas souvent celle de l'analyse géographique : l'appartement détaillé, l'immeuble et un ensemble restreint d'immeubles qui n'est même pas le quartier. Mais surtout le problème a été dépassé par la rediscussion permanente des catégories d'analyse et l'élaboration d'un vocabulaire spécifique et commun. La pratique interdisciplinaire oblige à acquérir une distance, elle contraint à des précautions dans l'usage d'un vocabulaire trop spontanément employé comme "politique de la ville * " contrôle des espèces urbaines ". Elle conduit à déconstruire un vocabulaire qui semble aller de soi comme le concept de ville, de qualité de vie en ville (Tobélem-Zanin, 1995), débouchant aussi, ce qui revient au premier point, sur une meilleure prise en compte de I'" homme - ou de l'" urbain - qui devient I'habitant, l'occupant de certains types d'appartements inscrit dans un paysage précis qui rend le qualificatif d'urbain quelque peu dérisoire.

Les concepts de milieu et de nature sont également de faux amis. Le milieu pour les écologues de l'équipe est défini à une échelle très limitée, celle de la niche écologique de l'espèce, de son habitat. II comprend un certain nombre d'éléments naturels comme le - climat - sans saisons des appartements. Mais ce qui est * naturel - pour les éthologistes, ce sont non seulement les blattes, mais aussi les habitants et même les conditions matérielles dans lesquelles se déploient les blattes en particulier, les sources d'eau, de lumière et de chaleur, les meubles etc. Également en quête du sens à donner au concept de milieu (dans la définition retrouvée de $P$. et $G$. Pinchemel (1989), de milieu urbain en particulier, ainsi que de celui de nature qui a motivé leur implication dans ce programme, les géographes constatent que leur notion de milieu ne va pas jusqu'à l'échelle de l'appartement, que le végétal ne fait pas partie du - milieu écologique = de cette espèce urbaine, et qu'ils n'utiliseraient pas le terme de microclimat à l'échelle de la cuisine et du palier des appartements.

La confrontation des usages de ces mots au sein de ces deux disciplines révèle là encore l'importance des échelles dans la définition de la notion de milieu, et souligne l'intérêt d'affiner ce concept par rapport à l'homme-habitant. Elle confirme les deux dimensions d'analyse des * natures de la ville - (Blanc, Mathieu, 1996) : la matérialité de la nature appréhendée différemment dans les deux disciplines, mais dont les emboîtements sont précisés ensemble, par exemple sur le thème des * saisons dans la ville - (Rivault, $1994)$; les représentations de la nature et de l'animal (désiré ou non désiré) dont la diversité explique en partie les différences de comportements des locataires par rapport aux blattes. Ces décalages de sens sont surmontés d'abord, comme nous l'avons déjà vu, par la remise en cause en commun de ces termes devenus stéréotypés, fausses passerelles entre les disciplines et les discours, transférés sans précaution d'un savoir à l'autre, débouchant trop facilement sur les conseils au gestionnaires, alors que la recherche n'a pas identifié et travaillé l'ensemble des segments qui permettraient d'énoncer la relation entre les dynamiques des deux populations (les hommes et les blattes) et les solutions à ce problème d'environnement. Mais la pratique interdisciplinaire a permis d'aller plus loin, jusqu'à la recherche faite en commun de ce qui peut être énoncé avec exactitude et de manière adéquate par chacune des disciplines (espèce urbaine pour les écologues, mode d'habiter en HLM ou milieu des grands ensembles pour les géographes), mais aussi conjointement (nature
7 À leur arrivée sur le - terrain - des écologues, les géographes ont constaté l'ampleur potentielle de leurs connaissances de type sociologique du fait des nombreuses visites et entretiens menés avant et pendant chaque désinsectisation. Mais cette connaissance n'était pas considérée comme - scientifique - parce qu'elle ne pouvait être schématisée (les locataires étaient identifiés par le numéro de l'appartement et le signe mâle ou femelle).

${ }^{8}$ Le terme de cafard est utilisé par tous les locataires pour désigner la - chose ", la - bête 1 - insecte - que les - dames-cafard - (nom donné par les locataires aux écologues) appellent du nom de blattes.

9 II n'est pas inutile de faire remarquer que les problèmes de pouvoirs, qui ont marqué l'expérience de certains chercheurs dans d'autres programmes interdisciplinaires ont été entièrement absents de celle-ci.

${ }^{10}$ II faut à cet égard rappeler qu'une des raisons de l'engagement des géographes dans cette recherche tenait au fait que, au colloque de Mions. les éthologistes avaient utilisé certains des concepts de la géographie, comme les termes d'urbain, populations urbaines, villes, habitat, logement, et même écologie urbaine, etc., ce qui ne pouvait que les intriguer. 
animale, pratiques de contrôle, locataires, politiques de désinsectisation, etc.). Peu à peu se sont dégagés les questions et termes utiles à l'articulation entre ces disciplines, au traitement de la question posée : représentations de la nature, de l'animal, pratiques de contrôle de la blatte, milieux...

\section{La valeur heuristique de l'interdisciplinarité}

La mise à plat de ces deux types de problèmes et l'exposé des moyens utilisés pour les surmonter illustrent déjà les résultats positifs de l'interdisciplinarité. II s'agit bien d'une progression scientifique dans la mesure où le dialogue interdisciplinaire oblige à plus de rigueur dans la méthode, à la reprécision, voire à la redéfinition des concepts utiles, à l'identification des spécificités et des intersections entre les disciplines concernées. Mais l'apport de l'interdisciplinarité à la recherche va encore plus loin que ces retombées théoriques et méthodologiques. Elle a une valeur heuristique dans la mesure où certains des résultats n'auraient pas pu être obtenus sans la pratique interdisciplinaire. On choisira, pour en témoigner, trois exemples : le premier fait plutôt progresser les questions de l'écologie, le deuxième est plus pertinent pour la géographie, le troisième constitue la réponse à la question finalisée - comment améliorer les politiques de désinsectisation ? -, qui intéresse plus particulièrement les services de santé des villes comportant des " grands ensembles .

${ }^{12} \mathrm{Cf}$. I'Ura Modélisation et traitements graphiques (CNRS/université de Rouen) dirigée par Yves Guermond, et Bussi $\mathrm{M}$. (1996). - Analyse spatiale de la consommation de médicaments

psychotropes : l'exemple

de l'agglomération

rouennaise *, Natures

Sciences Sociétés 4, 1, 37 -

49

13 Comme par exemple Jean-Paul Ferrier qui place au centre de sa réflexion le rapport des individus au territoire, la

- géographicité - de chaque individu, et privilégie l'espace de la quotidienneté.

14 Le plus loin que lion puisse aller dans ce sens est représenté par l'analyse des pratiques de l'espace en milieu urbain, c'est-à-dire les déplacements des individus et leur perception et

identification aux lieux. mais la maison.

l'appartement ne sont plus considérés comme des objets géographiques.

\section{Du côté de l'éthoécologie : une avancée dans la définition de la relation homme-blatte}

A travers la question - comment améliorer l'efficacité des désinsectisations et la qualité de vie des habitants des trois tours de la Zup ? ", les éthologistes cherchaient à réintroduire l'homme qu'on dit être exclu de l'écologie (Lefeuvre, Barnaud, 1992), ne serait-ce que parce que la population animale dont la dynamique est suivie est une espèce urbaine et que sa présence ou son absence dans des appartements habités par des hommes est une donnée essentielle du problème. Mais cette ambition était au départ peu précise et plutôt abstraite dans la mesure où l'écologue n'étudie pas habituellement l'homme, ni à fortiori la relation entre l'Homme (avec une capitale) et une population animale. La volonté simpliste de réintroduire l'homme dans l'éthologie d'une espèce urbaine s'est largement transformée sous l'effet des pratiques interdisciplinaires. Elle est devenue un autre ensemble de questions : i) comment ne pas traiter l'homme comme une boîte noire ? ii) comment appréhender les intersections entre les dynamiques de ces deux populations (les blattes et les habitants des HLM) ? iii) comment désigner théoriquement cette relation hommeanimal ?

Les biologistes proposent d'analyser cette relation comme une relation interspécifique entre deux espèces qui occupent les mêmes lieux, les géographes étant supposés connaître l'espèce humaine. Mais ces derniers, qui ne voyaient dans la blatte qu'un indicateur éventuellement pertinent des relations sociétés-nature en milieu urbain, ne sont pas disposés à traiter les habitants des HLM comme une espèce et refusent l'analogie. Pourtant, les entretiens révèlent qu'il s'agit bien d'une relation, et que celle-ci s'établit parce qu'il s'agit des mêmes lieux : la blatte est une intruse pour les habitants qui souhaitent unanimement et par dégoût sa destruction : quant à l'animal, il évite les moments et les lieux de risque, fuit à l'approche d'une présence humaine dangereuse. Les représentations du cafard et les pratiques qu'il provoque sont bien l'expression de relations entre ces deux types d'êtres vivants. À ces questions générales - comment peut-on définir la place de l'homme-habitant dans cette relation ? comment le biologiste peut-il intégrer l'homme dans l'étude de la dynamique des populations de blattes ? -, se substitue peu à peu une réponse qui ne provient ni de la géoanthropologie, ni de l'écoéthologie mais d'un entre-deux. Irons-nous jusqu'à dire, même si nous avons retrouvé des positions de l'ethnoscience, qu'une nouvelle approche de la relation homme-animal s'est dessinée.

La relation entre les deux disciplines a permis de penser que la relation homme-blatte ne peut être vue du seul point de vue biologique. Elle a permis de comprendre que l'histoire de l'espèce animale n'est pas séparable de l'histoire de l'appartement et de ses occupants " humains ", de leurs relations avec les voisins mais aussi de leur statut dans la société locale et globale. Cette relation n'est pas non plus séparable de l'histoire de l'immeuble, de ses gestionnaires, des déménagements et des installations, de l'état des espaces collectifs du dedans (la cage d'escalier, le vide-ordures, l'ascenseur) et du dehors (la façade, la pelouse, les chemins, les arbres).

Du seul point de vue biologique, cette relation ne peut pas être définie comme une relation hôte-parasite, relation oủ la blatte vivrait aux dépens de l'organisme humain. Mais les attitudes de rejet, de dégoût, voire de honte que cet animal suscite montrent que du point de vue des représentations, nous sommes assez proches de cette définition. Toujours du point de vue biologique, il ne s'agit pas non plus d'une relation prédateur-proie, relation oủ l'humain chasserait la blatte pour se nourrir, ni d'une relation mutualiste (symbiotique), oủ l'humain et la blatte auraient un avantage mutuel à vivre ensemble. Mais les chercheurs de sciences sociales ont montré que, chez certains habitants, la présence de cafards déclenche un comportement de chasseur à base de savoir naturel et de ruse. La mise en évidence de ce comportement a paru essentiel pour les biologistes, car il est potentiellement porteur de contrôle de la dynamique des populations de blattes.

Ce n'est pas non plus une relation de type commensal, où seule la blatte tire parti de la relation symbiotique, sans déranger l'humain. La blatte commensale détournerait la nourriture de l'homme à son profit, se ferait véhiculer par lui, ou bien tirerait protection de lui sans que sa présence le dérange ou 
le perturbe. Les blattes bénéficient certes de l'association avec l'homme, mais elles déclenchent de la part des habitants des réactions souvent violentes de répulsion dues en particulier à leur présence sur la nourriture et les instruments de cuisine, d'où des pratiques complètement orientées et modifiées par leur présence (lavage systématique de la vaisselle propre avant de s'en servir, appel à désinsectisation sans présence de blattes...).

Les blattes ne peuvent pas non plus être qualifiées d'espèce nuisible, c'est-à-dire entrant en compétition sur des ressources, provoquant des dégâts chiffrables sur les biens et les propriétés. Cependant, dans les représentations, la présence des blattes nuit à la valeur de l'appartement ; elle est associée à la mauvaise gestion collective des immeubles (exigence de rénovation). Imputée à l'autre, aux voisins, à l'étranger, cette présence devient le signe d'une nuisance véritable, celle du locataire voisin, avec ses blattes réelles ou imaginaires, qui empiète sur le territoire qui vous appartient.

Les blattes ne peuvent pas non plus être qualifiées d'espèce domestique, elles seraient alors élevées et sélectionnées par I'humain dans un objectif précis. Dans les représentations des habitants, le cafard est d'autant moins un animal domestique qu'il n'est pour ainsi dire jamais considéré comme un animal et même rarement comme un insecte. Pourtant la conscience que ces - bestioles - sont des espèces d'appartement, si ce n'est de maison, est générale, induisant, pour une minorité, des pratiques relativement laxistes comme s'il s'agissait d'un animal familier.

En définitive, les définitions classiques de la biologie ne permettent pas de qualifier la relation Homme-blatte. L'interdisciplinarité a transformé la question de départ. La notion d'Homme (avec une capitale) a perdu sa pertinence puisqu'il s'agit de relations d'une population précise donnée, voire d'individus hommes-habitants avec la population des blattes. La construction progressive de la notion de " pratiques de contrôle ", la priorité accordée à ce concept - mixte $=11$, mis en relation avec celui de - représentations individuelles et sociales *, ont permis de montrer qu'il existe des modèles de relation entre les habitants de ces trois tours et l'espèce Blattella germanica qui s'y est implantée. Ce * modèle habitant-blatte * pourrait avoir valeur générale non seulement parce qu'il énonce les relations entre individus et groupes sociaux et la nature animale, mais aussi parce qu'il renvoie aux rapports qu'entretiennent les - urbains - à leur habitat et à la * nature urbaine .

\section{Du côté de la géographie : la pertinence de la relation homme/animal pour interroger les rapports à la nature dans la ville}

II y avait, on l'a vu, un pari dans l'acceptation de cette recherche par les chercheurs géographes, alors même que cette discipline est aujourd'hui dominée en France par le paradigme de l'analyse spatiale : la géographie est la science de l'espace, de la ligne, du point, de la surface ; même si ses analyses convoquent le concept de représentations, elle doit se limiter aux * représen- tations spatiales * (Bailly, 1985), à l'* espace vécu * alors même que l'idée d'une ré-articulation de la géographie physique avec la géographie humaine (Mathieu, 1992) est contestée dans la mesure oủ elle substitue la notion de milieu à celle d'espace et redonne une place à la géographie physique dans une science qui s'affirme comme une science sociale : alors même enfin que la géographie urbaine s'intéresse parcimonieusement aux problèmes d'environnement, et surtout ne pose jamais la question de la nature dans la ville.

La géographie urbaine à ses débuts a certes pris en compte le site et la situation de la ville (topographie, collines, fleuve, climat et microclimat...) comme des éléments déterminants dans la genèse et l'évolution de la ville, assimilée alors à un organisme, à une individualité vivante. Mais elle s'est éloignée de ces premières études et ne fait plus intervenir la dimension naturelle du milieu urbain (Blanc, 1996). Aujourd'hui, même si certains manuels de géographie urbaine principalement destinés aux urbanistes, même si certains groupes de recherche ${ }^{12}$ tentent d'intégrer, dans l'analyse de la ville en général ou de telle ville particulière, les aspects physiques de problèmes d'environnement (pollution de l'air, espaces verts, ressources en eau), la géographie urbaine se préoccupe peu des rapports entre les représentations de la nature dans les villes et les natures matérielles de la ville (Tobelem-Zanin, 1995), et, a fortiori, des rapports sémantiques entre nature et environnement.

Entrer dans la question des rapports à la nature en ville, par l'animal, voire par le rapport des individushabitants à l'animal, est encore plus osé. L'hérésie est double. Parce que d'une part, côté géographie humaine, on fait peu de place à l'analyse des relations des individus et des petits groupes à l'espace, au territoire, et au milieu. À quelques exceptions près ${ }^{13}$, les géographes ont abandonné la microgéographie, l'analyse locale des rapports aux lieux ${ }^{14}$, les rapports sociétés locales-natures au point que la géographie rurale dont c'est une des spécificités - et des richesses - s'est trouvée injustement déqualifiée aux yeux de la géographie dominante.

Parce que d'autre part, la géographie physique, qui traite du milieu naturel et de l'environnement, a essentiellement abordé les aspects inertes de la nature, le substrat physique, l'étendue et ses formes, c'est-à-dire les éléments non vivants, la terre, l'air et l'eau. Certes la végétation joue un rôle important en biogéographie et dans les recherches sur le paysage. Mais les espèces végétales et animales ne font partie de la discipline que dans la mesure où elles peuvent être analysées comme structurant l'étendue terrestre, et alors constituent une forme, ou comme ressources (troupeau, pêche...). La géographie ignore en quelque sorte le vivant dans l'animal, ne s'intéresse pas aux rapports homme-nature animale.

Étudier les blattes urbaines manifeste bien un parti pris pour une géographie sans frontières, sans limites, qui se porte sur les thèmes et les objets qu'elle considère comme de son ressort, ou sur lesquels elle a quelque chose à dire ou envie de dire quelque chose. II se trouve d'ailleurs que chez les jeunes géné- 
rations de géographes (Blanc, 1996), la volonté de penser la relation entre écologie et géographie, entre le vivant et l'espace, est une préoccupation affirmée. II en est de même de la relation entre anthropologie et géographie qui a été essentielle dans cette recherche.

Or, entrer dans la géographie urbaine, et par la nature, et par l'animal, s'est avéré pertinent, cette approche permettant même d'avancer quelques bases théoriques dans la recherche sur les problèmes d'environnement urbain. En effet la blatte est une espèce exemplaire pour clarifier la double dimension des rapports sociétés-natures, rapports à la nature en tant qu'elle est matérialité (avec une logique de - fonctionnement " qui lui est propre), rapports à la nature en tant qu'elle est culture. La naturalité des blattes, celle que les écologues décrivent et observent, rappelle et corrobore, plus encore que celle des arbres et des plantes, la présence (au sens fort) de systèmes naturels dans la ville, à toutes les échelles. L'espèce blatte fait objectivement partie de la nature urbaine puisqu'elle s'adapte au climat de l'immeuble " sans saisons ", partage I'habitat du " ménage ", envahit différents espaces urbains dans la limite de son écologie propre, pose des problèmes de contrôle et de maîtrise aux individus comme aux collectivités locales. Elle introduit à la définition de ce que l'on pourrait nommer * objet naturel urbain *.

Quant à l'analyse des représentations du * cafard *, elle confirme l'existence, dans l'imaginaire des habitants ordinaires comme dans les représentations savantes, d'une opposition entre l'idée de nature (exclusivement associée à la campagne) et l'idée de ville (lieu d'artifice et de maîtrise, lieu technique). Ce qui distingue l'animal, et encore plus la blatte, de ce que les enquêtés associent à l'idée de nature, c'est sa mobilité instantanée. Avec une vélocité plus grande que d'autres espèces animales présentes en ville, la locomotion de cet insecte de petite taille est à l'origine de la représentation des maux qu'elle engendre : envahissement invisible, déplacement d'un appartement à l'autre, provenance étrangère... La difficulté de lui attribuer une place, de contrôler ses déplacements, explique que l'animal n'est pas considéré comme naturel dans les représentations de l'habitant citadin. La nature en ville ne peut être que désirée ou maîtrisée ; les natures de la ville ne peuvent être que niées ou contrôlées. Dans l'espace urbain, et en particulier dans l'espace du logement, le naturel n'a d'existence naturelle que s'il est désiré, identifié, contrôlé, s'il est un naturel produit.

L'animal en ville ne structure donc pas l'espace, mais il informe avec acuité sur la relation paradoxale des habitants à leur environnement naturel. II permet de comprendre pourquoi, dans sa dimension idéelle, la nature ne peut exister dans la ville non seulement parce que ces termes sont culturellement antinomiques, mais parce que cette antinomie induit un effacement de la nature dans la pratique et la politique de la ville (Blanc, Mathieu, 1996). L'hypothèse d'une nécessaire identification des * cultures de la nature - des individus-habitants pour progresser dans la question de I* environnement urbain " se trouve confirmée.
L'entrée par l'animal fait aussi redécouvrir aux géographes de l'équipe le concept d'habiter (Sivignon, 1993), de mode d'habiter comme concept mixte, qui réactive et concrétise pour la géographie celui de milieu (Pinchemel, 1989) si difficile à mettre en œuvre, et qui pourtant est le plus proche des relations sociétés-natures. Ce concept est aussi proche de celui de genre de vie proposé par Maximilien Sorre (Sorre, 1952) qui y inclut tous les éléments de la vie des individus et des groupes allant jusqu'à affirmer que des groupes transportent leurs genres de vie. Étudier I'animal en ville permet au géographe de traiter le milieu urbain en tant qu'il associe dans les représentations et pratiques du citadin différents niveaux de l'habiter (de l'appartement à la ville) ouvrant de nouvelles voies pour l'analyse géographique de la ville comme milieu de vie. Lanimalité des blattes, comme nature incontrôlée, associée au dégoût et à la répulsion, renvoie plus largement à la gestion des milieux urbains. S'impose donc l'idée que la réintroduction de la dimension naturelle de la ville à travers le concept de mode d'habiter ne peut se passer de la relation à l'animal même si d'autres rapports sont à étudier. La géographie d'aujourd'hui retrouve, ce faisant, la veine audacieuse et holistique (affrontant toutes les questions) des années de la " géographie établie - (Pinchemel et al., 1984).

\section{Des deux côtés, une réponse circonstanciée à la question finalisée}

\begin{abstract}
À la question opérationnelle qui était à l'origine de cette recherche - comment réduire la pullulation des blattes dans des immeubles collectifs et comment améliorer le contrôle de la dynamique de cette espèce ? -, la recherche a apporté des réponses originales validées tantôt dans le cadre des disciplines, tantôt grâce à l'interdisciplinarité. Côté écologie, certaines idées reçues sur lesquelles sont en partie fondées les décisions d'intervention dans les immeubles, se sont avérées fausses. II en est ainsi de la corrélation entre saleté des appartements et pullulation, entre présence d'étrangers et familles nombreuses et présence de blattes. La mise en rapport des densités de blattes observées après les désinsectisations avec les caractéristiques des locataires et des appartements, montre que la variable présence/ absence de blattes varie indépendamment de la variable propreté (cf. encadré 5), que la pullulation n'est pas liée à une occupation par des étrangers.

II a été aussi démontré que l'amélioration de l'efficacité des désinsectisations n'est pas un problème qui se résoudra par la seule expertise. Le recours à des spécialistes de l'écologie des blattes, ou à des experts en produits chimiques adaptés à cette espèce, ne suffiront pas pour renouveler les politiques * sanitaires - de la ville. Cette position, qui était hypothèse implicite chez les éthologistes au départ de la recherche, est devenue explicite et évidente après l'enquête des géographes. On pouvait répondre aux
\end{abstract}


gestionnaires des HLM qu'il était inutile de multiplier ou de réduire les campagnes de désinsectisations si on n'accordait pas la priorité à la connaissance des représentations des habitants et de leur relation avec les pratiques de contrôle.

La mise en place de stratégies de lutte contre la pullulation des blattes doit d'abord tenir compte des raisons pour lesquelles certaines personnes n'ouvrent jamais leur porte. Le refus systématique des désinsectisations s'explique surtout parce que la présence de * cafards - est le signe d'une stigmatisation sociale, de la non-réussite. Mais, ne pas ouvrir sa porte, c'est aussi l'expression d'un refus de l'ingérence chez soi, du propriétaire, des * autres ", surtout quand il y a " malheur - ou * misère - à cacher. Or si la densité de blattes est forte, ces appartements offrent un risque maximum de rediffusion des blattes après désinsectisation. Les représentations et comportements de cette catégorie de locataires sont donc particulièrement importantes à approfondir pour résoudre le problème d'ensemble.

La même crainte d'une dévalorisation sociale produit chez certaines personnes un comportement inverse. Alors qu'il n'y a pas de blattes dans leur appartement, elles emploient abondamment des insecticides dangereux pour la santé, acceptent toutes les désinsectisations * inutiles = pour montrer que, contrairement aux autres, elles sont maitres de leur intérieur. Ces pratiques ne contribuent en rien à la réussite des objectifs collectifs de la désinsectisation et créent des risques sanitaires superflus.

Les politiques de désinsectisation doivent aussi tenir compte du fait que la présence de blattes est généralement associée au sentiment d'inconfort qu'engendrent les fortes densités humaines et l'habitat social. Elle suscite des réactions qui relèvent de l'idéologie (idée de saleté, rejet des immigrés, refus de la vie en immeuble collectif) mais aussi d'une intelligence du réel (inconfort et mauvaise qualité de la vie, problèmes d'hygiène, risques pour la santé, allergies). Les pratiques de contrôle sont très variées : utilisation intensive d'insecticide, division sexuelle de l'* écrasement " de l'animal, destruction sélective et scientifique, destruction anarchique, tolérance de fait, etc. Les rapports familiaux (la femme attend I'homme pour tuer I'animal), l'attachement au logement (importance de l'investissement affectif), les rapports aux voisins et à l'espace collectif (qualité de l'entretien, critique de la gestion), sont les fondements de cette diversité. Dans la mesure oủ le contrôle de la pullulation suppose le contrôle dans chaque appartement de l'immeuble, l'ensemble des pratiques de contrôle doit être connu. Les rapports entre voisins jouent un rôle particulièrement important pour définir les différentes attitudes. Leur connaissance est indispensable pour proposer des solutions collectives à l'échelle de l'immeuble. Celles-ci supposent un travail d'information et d'animation, intégrant les apports de cette recherche et la mise au point d'un protocole comportant des règles biologiques et sociologiques à l'échelle de chaque immeuble. Sous ces conditions, il est possible de développer les connaissances individuelles de chaque - ménage - et de faire accepter à tous le fait qu'il existe une relation entre ce qui se passe dans son espace privé, dans l'espace * intime * de son chez soi, et ce qui se passe dans l'immeuble collectif.

En conclusion on reviendra sur ce qui a compté dans cette expérience interdisciplinaire. C'est avant tout d'avoir pu prouver que le dialogue entre sciences sociales et sciences biologiques, au demeurant assez rare, est possible et fructueux. C'est ensuite d'avoir révélé l'intérêt de repenser la question de la nature dans la ville en se dégageant de l'association traditionnelle entre qualité de vie en ville et espaces verts, en montrant que l'animal est un indicateur pertinent pour évaluer la qualité du cadre de vie, en établissant un modèle d'analyse des relations nature/citadins étayé sur le concept de mode d'habiter. C'est aussi de témoigner que la pratique interdisciplinaire est compatible avec la progression disciplinaire, chaque discipline trouvant avantage à revenir sur sa préoccupation spécifique. C'est enfin d'avoir confirmé que la nécessité de l'action était le fondement * impérieux * de l'interdisciplinarité, de même que l'acceptation par tous les partenaires de la finalisation d'une recherche en est incontestablement la clé la plus importante de réussite.

Mais on peut aussi poser la question de sa reproductibilité. Nous avons tenté de donner les ressorts objectifs d'une collaboration réussie : choix délibéré de l'interdisciplinarité, identification précise des problèmes et des intersections entre les disciplines, rigueur de la réflexion sur les concepts et les outils, volonté de répondre à la question posée. Mais audelà de ces critères, ne doit-on pas souligner l'importance de caractères subjectifs liés aux personnalités des chercheurs qui s'associent dans une opération à durée limitée ? Quelques remarques ont été soulignées en commun : nous étions quatre partenaires de même sexe, oubliant nos différences de statut et de rang, refusant les rapports de pouvoirs, la rivalité, les pratiques hiérarchiques : rencontres et échanges fréquents comportant des temps hors-travail de convivialité ont jalonné cette recherche ; nous avons accepté de travailler sur un objet commun sans chercher à privilégier notre qualité, notre spécificité, en nous mettant au service de la question posée ; nous avons opté pour une écriture collective de nos résultats en acceptant de donner un rôle moteur au partenaire de sciences sociales le plus à même de rédiger, de formaliser le dialogue ; nous affirmons l'importance du consensus sur ce qui est écrit, l'écriture interdisciplinaire d'une recherche étant, malgré sa difficulté, la meilleure preuve de son achèvement.

Résumé : Cet article concerne une expérience
interdisciplinaire limitée à deux disciplines et portant sur
une question finalisée : comment améliorer le contrôle
d'une espèce urbaine Blattella germanica dont la
pullulation dans les immeubles collectifs contribue au mal-
être des habitants. Le retour critique sur cette expérience a
un triple intérêt : repérer les problèmes généraux que
pose la pratique interdisciplinaire ; approfondir le
problème particulier des relations entre géographie et
écologie animale ; établir le bilan des résultats spécifiques
de cette interdisciplinarité rendue aisée par la réussite de
lexpérience.




\section{RÉFÉRENCES}

Bailly A. (1985). Distances et espaces : 20 ans de géographie des représentations - L'espace géographique 3, 197-205

Blanc N. (1996). - La nature dans la cité •, thèse de doctorat (sous la direction de N. Mathieu), université de Paris-1, $400 \mathrm{p}$.

Blanc N., Mathieu N., Cloarec A., Rivault C. (1993). - La finalisation, facteur positif de la pratique interdisciplinaire : les blattes urbaines : communication aux journées de Natures Sciences Sociétés: Dialogues, 13 p. + annexes

Blanc N., Mathieu N. (1996). - Repenser l'effacement de la nature dans la ville • in Villes, Cities, Ciudades, Le courrier du CNRS 82, 105-107

Digard J.P. (1990). Lhomme et les animaux domestiques. Paris, Fayard, coll. · Le temps des sciences $\cdot, 325 \mathrm{p}$.

Guillerme A. (1990). Le temps de l'eau, la cité, l'eau et les techniques. Seyssel, Champvallon, coll. $\cdot$ Milieux $\cdot, 263 \mathrm{p}$.

Guillerme A. (1994). - La disparition des saisons dans la ville - Les annales de la recherche urbaine $61,9-15$

Jollivet M. (dir.) (1992). Les passeurs de frontieres, Sciences de la nature, sciences de la société. CNRS, Paris, $589 \mathrm{p}$.

Jollivet M., Pavé A. (1993). • L'environnement un champ de recherche en formation *: Natures Sciences Societés 1, 1, 6-21

Lefeuvre J.-C., Barnaud G. (1992). - Lécologie avec ou sans Ihomme ? •, in Sciences de la nature, sciences de la societé, les passeurs de frontières. CNRS Paris, 70-112

Legay J.-M. (1986). - Quelques réflexions à propos d'écologie : défense de I'indisciplinarité - Acta Oecologica, Oeologica Generalis 7, 4, 391-398

Lévy J.-C. (1992). - La ville dans la nature : quelles recherches sur l'environnement urbain ? : Actes du colloque national d'écologie urbaine, 27-28 septembre 1991, Mions (Universite C.-Bernard-Lyon-I, ed.), Villeurbanne, 220-225

Lévy J.C. (1993). - Ville, environnement et aménagement territorial · Reed. $43-44,3-9$

Mathieu N. (1987). - L'interdisciplinarité en question dans l'observatoire Causses-Cévennes - Actes du colloque - Valorisation et maitrise du territoire dans les montagnes sèches de l'Europe du Sud :, Alès, 20 sept.-1er oct 1987), BSLG 21, 3-4, 493-504

Mathieu N. (1989). - Démarches interdisciplinaires et perspectives d'avenir bilan de 25 ans de pratique $\cdot$, in : Interdisciplinarité zones marginales et France du Sud (Actes du colloque du 17-18 novembre 1988), Marseille Piren/CNRS/Cres/Cams/EHESS

Mathieu N. (1992). - L'environnement dans la géographie urbaine actuelle diagnostic et perspectives : Actes du colloque national d'écologie urbaine, 27-28 septembre 1991, Mions (Université C.-Bernard-Lyon-I, ed.) Villeurbanne, 124-131

Mathieu N. (1992). - Céographie et interdisciplinarité : rapport naturel ou rapport interdit ? - In : Sciences de la nature sciences de la societe. Les passeurs de frontieres, CNRS, Paris, 130-154

Mathieu N. (1997). - Rural et urbain : unité et diversité dans les évolutions des modes dhabiter * in : LEurope et ses campagnes (M. Jollivet, dir.), Presses FNSP, 151-173

Mathieu N., Jollivet M. (1989). Du rural a l'environnement : la question de lo nature aujourd hui. Arf, LHarmattan, Paris, $352 \mathrm{p}$.

Micoud A. (1993). - Vers un nouvel animal sauvage : le sauvage "naturalisé vivant" ? : Natures, Sciences, Sociêtés 1, 3, 202-210

Morin E. (1996). - Transdisciplinarité, interdisciplinarité, le dialogue est-il possible ? (un entretien avec Edgar Morin, propos recueillis par N. Mathieu, A. Pena, M. Cohen, texte établi par A. Auris), Natures Sciences Sociétés 4, 3, 250-257

Rivault C., Cloarec A. - Limits of insectide cockroach control in council flats in France'. J. Environnement Management 45, 379-393

Rivault C., Mathieu N., Cloarec A., Blanc N. (1994). • Les blattes, Un animal de ville à toutes saisons : Les Annales de la recherche urbaine 61, 31-37

Rivault C. (dir)., Cloarec A., Mathieu N., Blanc N. (1995). Les blattes en milieu urbain. CNRS, Rennes; Strates, Paris, $100 \mathrm{p}$.

Pinchemel P., Robic M.-C., Tissier J.-L (1984). Deux siècles de géographie francaise. Choix de textes. CTHS, Paris

Pinchemel P. et G. (1989). La face de la terre. Armand Colin, Paris, 519 p.

Pontier D., Yoccoz N. (1992). - Vertébrés des villes, vertébrés des champs : Intérêt d'une écologie des populations urbaines . Actes du colloque national d'écologie urbaine, 27-28 septembre 1991, Mions (université C. Bernard-Lyon-l, ed.), Villeurbanne, 132-134

Robic M.C. (dir.) (1992). Du milieu à l'environnement. Économica, Paris, $200 \mathrm{p}$

Sivignon M. (1993). - Du verbe habiter et de son amère actualité: Revue géographique de Lyon $68,4,6-14$

Sorre M. (1952). Les fondements de la geographie humaine. Tome 3. Lhabitat et conclusion générale. Armand Colin, Paris, $499 \mathrm{p}$.

Tobelem-Zanin C. (1995). La qualité de la vie dans les villes françaises. Université de Rouen, collection - Nouvelles Donnes en géographie *, Rouen, $288 \mathrm{p}$.

Vourch A., Pelosse V. (1988). Chasser en Cévennes: un jeu avec l'animal. Edisud Paris

Vourch A. (1990). - Représentation de l'animal et perception sociale de sa réintroduction. Le cas du lynx des Vosges : La terre et la vie, suppl. 5 . $175-187$ 